\title{
Limonin inhibits angiogenesis and metastasis of human breast cancer cells by suppressing the VEGFR2/IGFR1-mediated STAT3 signaling pathway
}

\author{
Jing Chen ${ }^{1,2 \#}$, Bo-Xia Liu ${ }^{1,2 \#}$, Qin Shen ${ }^{1,2}, \mathrm{Na} \mathrm{Li}^{1,2}$, Jun Ling ${ }^{1,2}$, Min Xiao ${ }^{3}$, Hai-Yan Jiao ${ }^{1,2}$, Tao Li ${ }^{4}$ \\ ${ }^{1}$ School of Basic Medical Sciences, Ningxia Medical University, Yinchuan, China; ${ }^{2}$ Key Laboratory of Fertility Preservation and Maintenance \\ (Ningxia Medical University), Ministry of Education, Yinchuan, China; ${ }^{3}$ Qingpu Branch of Zhongshan Hospital, Fudan University, Shanghai, China; \\ ${ }^{4}$ Department of Oncology, General Hospital of the Ningxia Medical University, Yinchuan, China \\ Contributions: (I) Conception and design: BX Liu, T Li; (II) Administrative support: J Chen; (III) Provision of study materials or patients: Q Shen, \\ J Chen, HY Jiao; (IV) Collection and assembly of data: J Chen, BX Liu, Q Shen; (V) Data analysis and interpretation: BX Liu, Q Shen, J Ling, M \\ Xiao; (VI) Manuscript writing: All authors; (VII) Final approval of manuscript: All authors. \\ \#These authors contributed equally to this work. \\ Correspondence to: Prof. Tao Li; Prof. Haiyan Jiao. Department of Oncology, General Hospital of the Ningxia Medical University, 1160 Shengli Road, \\ Yinchuan 750004, China. Email: lit1979@163.com.
}

Background: Limonin is one of the major active ingredients of citrus. In the present study, the antiangiogenic and anti-metastatic effects of limonin were investigated.

Methods: The Molecular docking assay was carried out to assess the binding ability of limonin with VEGFR2 receptor. MTS assay was used to detect the effect of limonin on the proliferation of breast cancer cells (MDA-MB-231, MCF-7). The Wound-healing and Transwell chamber invasion assays were used to detect the inhibition effect of limonin on migration and invasion of HUVECs cells or breast cancer cells. The capillary-like tube formation assay and Matrixgel plug experiment were used to further measure the in vivo anti-angiogenic activity of limonin. Western blot, RNA isolation, microarray data analysis and RTPCR were used to explore the molecular mechanism of limonin in suppressing breast cancer angiogenesis and metastasis. Left ventricular tumor metastasis model and caudal vein tumor metastasis model of breast cancer were both applied to verify in vivo anti-metastatic effects.

Results: Limonin dose-dependently inhibited the vascular endothelial growth factor (VEGF)-mediated tyrosine phosphorylation of vascular endothelial growth factor receptor 2 (VEGFR2) by blocking VEGF binding to VEGFR2 and suppressing constitutive STAT3 activation in human umbilical vein endothelial cells. Limonin effectively inhibited VEGF-induced endothelial cell proliferation, migration and tubularstructure formation in vitro and markedly reduced VEGF-triggered neovascularization in mouse matrigel plugs in vivo. Moreover, limonin treatment led to a remarkable suppression of tumor metastasis by decreasing the phosphorylation of insulin growth factor receptor 1-mediated STAT3 and the expression levels of its downstream members MMP-9 and VEGF in breast cancer cells. The data further showed that limonin increased the levels of the negative STAT3 regulator SHP-1 in breast cancer cells.

Conclusions: Limonin is a promising anti-angiogenic and anti-metastatic candidate compound that can be further optimized as a therapeutic agent for breast cancer.

Keywords: Limonin; breast cancer; angiogenesis; metastasis; STAT3

Submitted May 04, 2020. Accepted for publication Sep 30, 2020.

doi: $10.21037 /$ tcr-20-1992

View this article at: http://dx.doi.org/10.21037/tcr-20-1992 


\section{Introduction}

Metastasis is a multi-step process. Cancer cells must migrate from the primary tumor, intravasate into the lymphatic and/or the circulation, evade immune surveillance, survive in the circulation and proliferate in remote organs $(1,2)$. During these processes, the initiation of angiogenesis plays a key role in the growth and spread of the tumor cells (3). Given that vascular endothelial growth factor receptor 2 (VEGFR2) plays a predominant role in promoting angiogenesis, more than 10 angiogenesis inhibitors that specifically target VEGFR2 have been used in the clinic against cancer. However, several issues can not be ignored and remain to be solved (4). These agents only offer very limited survival benefits and possibly enhance tumor aggressiveness by stimulating treatment resistance $(5,6)$. This indicates that the development of additional novel therapies for patients with metastasis is highly imperative $(5,6)$. Recent studies have shown that specific compounds exhibit a dual inhibition of angiogenesis and metastasis and are even more effective since they exert synergistic inhibitory effects on the metastatic process (7).

Citrus is widely used as a dietary supplement for both nutrition and health-promoting purposes $(8,9)$. Recent studies have shown that the most important bioactive molecules in citrus are carotenoids, flavonoids, and limonoids. Among them, limonin is one of the most bioactive components of the limonoid class that was initially reported to exert significant anti-schistosomal, anti-malarial, antiviral, anti-inflammatory, antioxidant and cholesterol-lowering activities (10-14). Subsequent studies have found that limonin exhibits anticancer potential via the induction of apoptosis in various human tumor cell lines $(15,16)$. Notably, limonin induces apoptosis of melanoma and osteosarcoma cells and of lung, colon, oral and breast cancer cells in vitro $(15,16)$. However, the effects of limonin on breast cancer progression have not been fully clarified.

In the present study, the anti-angiogenic and antimetastatic effects of limonin on human umbilical vein endothelial cells (HUVECs) and on the highly metastatic human breast cancer cells MDA-MB-231 and MCF-7 were investigated in vitro and in vivo. Moreover, the underlying molecular mechanisms were characterized. The findings indicate that limonin is a promising anti-angiogenic and anti-metastatic candidate compound that can be further optimized for breast cancer treatment. We present the following article in accordance with the ARRIVE reporting checklist (available at http://dx.doi.org/10.21037/tcr-201992).

\section{Methods}

\section{Chemicals and reagents}

Limonin (purity $>99.37 \%$ ) was purchased from Shanghai Winherb Medical Science. A $50 \mathrm{mmol} / \mathrm{L}$ stock solution was prepared in dimethyl sulfoxide (DMSO) (Sigma, St. Louis, MO), Regorafenib were purchased from Selleck Chemicals (Houston, TX). Growth factor-reduced Matrigel was purchased from BD Biosciences (San Diego, CA). Primary antibodies against phosphor-specific anti-VEGFR2 $\left(\mathrm{Tyr}^{1175}\right)$, VEGFR2, phospho-STAT3 $\left(\mathrm{Tyr}^{705}\right)$, STAT3, Bcl2, BCLxl, MMP-9 and SHP-1 were obtained from Cell Signaling Technology (Danvers, MA, USA; 1:1,000 dilution). VEGF (A-20), IGFR, phospho-IGFR(Tyr $\left.{ }^{1316}\right)$ was purchased from Santa Cruz Biotechnology (Santa Cruz, CA; 1:1,000 dilution). $\beta$-actin and GAPDH were obtained from Hangzhou Huaan Biotechnology Company(China; 1:1,000 dilution). Goat Anti-Rabbit IgG and HRP Goat Anti-Mouse IgG were provided from Abbkine (1:2,500 dilution). Anti-CD31 and anti-Ki67 were provided by Abcam (UK; 1:200 dilution). SiRNA and scrambled control was purchased from Santa Cruz Biotechnology (Santa Cruz, CA). Recombinant human VEGF $\left(\mathrm{VEGF}_{165}\right)$ was acquired from R\&D Systems (MN, USA). All other reagents were obtained from Sigma-Aldrich (St Louis, MO, USA).

\section{Cell culture}

Primary human umbilical vein endothelial cells (HUVECs) and the luciferase-labeled human mammary adenocarcinoma MDA-MB-231 cell lines were gifted from Dr Mingyao Liu (The Institute of Biomedical Sciences and School of Life Sciences, East China Normal University, Shanghai, China) and cultured in endothelial cell culture medium (ECM) as described previously $(17,18)$. The two human breast cancer cell lines, MCF-7 and MDA-MB-231, were from American Type Culture Collection. All these cells were maintained in a humidified atmosphere at $37{ }^{\circ} \mathrm{C}$ with $5 \% \mathrm{CO}_{2}$ using RPMI-1640 with $10 \%$ FBS.

\section{Molecular docking}

The docking assay was carried out to assess the binding ability of limonin with VEGFR2 receptor by using Autodock 4.2 as described previously (19). The ligands were drawn by using Chemoffice, and Openbabel without structural optimization imported ligand into 3D structure (20). 
The Gasteiger charges and hydrogen atoms were added to the ligand and receptor using the MGLTools1.5.6. The protein structure was obtained from Protein Data Bank (http://www.rcsb.org), and the next step was to remove water and salt ions. Docking Grid Box has size of $56 \AA^{*} 40 \AA^{*} 40 \AA$ and the centre of box was $(-27.76,-0.681$, $-8.054)$. The graphics of molecule-protein interaction was visualized and analyzed using UCSF Chimera1.9 and LigPlot with default parameter.

\section{Cell viability assay}

Briefly, HUVECs or cancer cells were incubated in 96well plate at density of $4.5 \times 10^{4}-5.5 \times 10^{4}$ cells per well and treated with or without VEGF $(10 \mathrm{ng} / \mathrm{mL})$ or increasing serial doses of limonin $(0-500 \mu \mathrm{M})$ for $72 \mathrm{~h}$. Thereafter, cell viability was determined using MTS kit as described previously $(17,18)$. All of the experiments were performed in three independent experiments.

\section{Wound-healing assay}

The assay was performed as described previously $(17,18)$. HUVECs or tumor cells were incubated in 6-well plates and treated serum starvation for $24 \mathrm{~h}$ before scratching. The cells were washed with PBS for three times, scratched with the $100 \mu \mathrm{L}$ tips and then incubated in growth medium with or without VEGF $(10 \mathrm{ng} / \mathrm{mL})$ or limonin at 40 and $60 \mu \mathrm{M}$. After cultured 8-24 h, photos were taken using an inverted microscope (TE2000, Nikon, Japan). The migrated cells were observed from three randomly selected regions and quantified by manual calculation. Inhibition percentage was expressed as a percentage of the vehicle control (100\%). All of the experiment were repeated independently at least three times.

\section{Transwell chamber invasion assay}

To check the impact of limonin on HUVECs cell motility and invasive ability in vitro, we performed cell migration assay using gelatin-coat Boyden inserts $(8-\mu \mathrm{m})$ (BD Biosciences) as reported previously $(17,18)$. The assays were performed for $8-10 \mathrm{~h}$ after $0.5 \mathrm{~h}$ of pre-treatment with or without VEGF ( $50 \mathrm{ng} / \mathrm{mL}$ ) or different doses of limonin (40 and $60 \mu \mathrm{M})$. Cells that migrated to the side were fixed and stained with $0.5 \%$ crystal violet and then observed by the optical microscope.

\section{Endothelial cell capillary-like tube formation assay}

The capillary-like tube formation assay was conducted as described before $(17,18)$. HUVECs $\left(7 \times 10^{4}\right.$ cells per well) were cultured in ECM and subjected to different concentrations of limonin for $30 \mathrm{~min}$, and then added to the matrigel and incubated at $37^{\circ} \mathrm{C}$ for $10 \mathrm{~h}$. The capillary-like morphogenesis of HUVECs in three random microscopic fields were examined using an inverted microscope (TE2000, Nikon, Japan) at $100 \times$ magnification. The numbers of branching nodes in three random fields per well were examined by manual counting. Cells in only ECM medium were served as a vehicle control. Each experiment was independently repeated at least three times.

\section{Matrigel plug assay}

Matrigel plug assay was performed as previously reported $(17,18), 500 \mu \mathrm{L}$ of Matrigel with or without $100 \mathrm{ng}$ of VEGF, 20 units of heparin, and representing quantities of limonin (200 and $400 \mu \mathrm{g}$ ) were subcutaneously injected into the ventral region of C57BL/6 mice $(n=3)$. Seven days after implantation, the complete basement membrane matrix plug was carefully removed and then fixed and embedded in the paraffin. Sections $(4 \mu \mathrm{m})$ were stained with indicted antibodies. Microphotographs were taken using an OLYMPUS BX41 photomicroscope (magnification at $400 \times$ ).

\section{RNA isolation and microarray data analysis}

MDA-MB-231 cells $\left(2 \times 10^{6}\right)$ were seeded in $10 \mathrm{~cm}$ cell culture dishes and then were treated with different limonin concentrations $(0,40,60 \mu \mathrm{M})$ for $24 \mathrm{~h}$. Cells were collected by using Trizol Reagent (Invitrogen Carlsbad, CA, USA), and mRNA microarray was analyzed according to specification by the Gene Tech Company Limited (Shanghai, China) by using Affymetrix Gene Chip Prime View Human Gene Expression array (Affymetrix). FatiGo of the Babelomics software was used to do the TFBS Enrichment Analysis. The differentially expressed genes (DFGs) were determined based on a false discovery rate threshold of 0.05 as previously reported (21).

\section{Western blotting analysis}

Western blots were performed as described previously 
$(17,18)$. Totally, $35 \mu \mathrm{g}$ of the cell lysates were separated on $8-12 \%$ SDS-PAGE and electrophoretically transferred to PVDF membranes. The membranes were blocked in $5 \%$ BSA, incubated with primary antibodies overnight at $4{ }^{\circ} \mathrm{C}$, and then followed by subsequent incubation with secondary antibodies. Immunoreactive bands was performed by an enhanced chemiluminescence (ECL).

\section{$R N A$ isolation and reverse transcription PCR}

Total RNA extracted from MDA-MB-231 cancer cells were treated with increasing serial doses of limonin for $24 \mathrm{~h}$ using the Trizol reagent. And cDNA was synthesized using a reverse transcriptase PCR (RT-PCR) kit (Thermo).

\section{Transfection with SHP-1 siRNA}

For siRNA-mediate knockdown of SHP-1 was performed as previously described (22). Briefly, MDA-MB-231 cells were seeded in a 6-well cell culture dish, then transfected with the SHP-1-siRNA or vehicle control for $24 \mathrm{~h}$ and used for subsequent experiments.

\section{In vivo mammary tumor metastasis assays}

All mouse experiments were carried out in accordance with the animal experimental regulations approved by the Animal Protection and Utilization Committee of Ningxia Medical University. All nude mice (6-8 weeks) were purchased from the Laboratory Animal Center of Ningxia Medical University and raised in SPF (Specific Pathogen Free) environment. Two kinds of mouse tumor metastasis models were established: left ventricular tumor metastasis model and caudal vein tumor metastasis model. The above models were divided into control group and limonin treatment group. Eight female BALB/c nude mice in each group were injected with MDA-MB-231-Luc cells $\left(1 \times 10^{6}\right)$ through tail vein and left ventricle respectively. One week before tail vein and left ventricular injection, vehicle or limonin $(25 \mathrm{mg} / \mathrm{kg} / \mathrm{d}$ ) was pretreated by intraperitoneal injection. The total experimental period of the former is 28 days, and the latter is 45 days. Drug intervention was carried out by intraperitoneal injection of limonin every other day. Tumorbearing mice are checked daily for their physical condition. During the experiment, no stress or abnormal behaviors due to tumor bearing were observed in the mice. Animals were placed onto the warmed stage inside the camera box and received continuous exposure to $2.5 \%$ isoflurane to sustain sedation during imaging. Every group of mice was imaged for $30 \mathrm{~s}$. IVIS detection system was used to image and quantify tumor metastasis. After the experiment, all mice were killed with excess amounts of anestheti and all efforts were made to minimize suffering. The tumor, heart, liver, spleen, lung and kidney were taken for further observation and study.

\section{Immunobistochemistry analysis}

IHC was performed as previously described protocols $(17,18)$. Immunohistochemistry on $5-\mu \mathrm{m}$ sections of paraffin-embedded specimens and staining with Ki-67, p-STAT3 and MMP9 antibodies. Images were gained from a Leica microscope (Leica, DM4000b).

\section{Statistical analysis}

Inter-group comparisons were performed by one-way analysis of variance and Dunnett's test. Data are expressed as mean $\pm \mathrm{SD} . \mathrm{P}<0.05$ was considered statistically significant.

\section{Results}

Limonin suppresses vascular endothelial growth factor (VEGF)/VEGFR2-mediated STAT3 signaling pathway by blocking VEGF binding to VEGFR2. The important role of the VEGF/VEGFR-2 axis in the regulation of endothelial mitogenesis and migration is widely accepted $(23,24)$. The molecular structure of limonin is shown in Figure $1 A$. In the present study, it was hypothesized that limonin may bind to VEGFR-2 and affect the interaction of VEGF with VEGFR-2. In order to confirm this hypothesis, the binding mode of the inactive conformational structure of limonin to VEGFR-2 was analysed by molecular docking. The results indicated that limonin could occupy the activity pocket by interacting with specific amino acid residues by hydrophobic or hydrophilic interactions. The amino acid residues Va1914, Leu889, Ile888, Arg1027, Ile892, His1026 and Asp1046 interacted with the protein by hydrophobic interactions (Figure 1B,C). In addition, the amino acid residues Glu885, Lys868, Cys1045, Val899, Ile1044, Ile1025 and Asp814 interacted with the protein through hydrophilic interactions (Figure 1B,C). Among them, K868, V899 and D1046 residues interacted with adenosine triphosphate (ATP) and limonin through different interaction patterns (Figure 1D). Furthermore, 
A

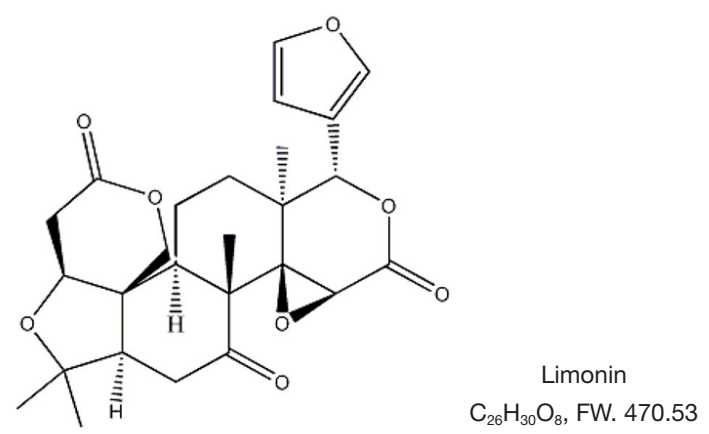

B

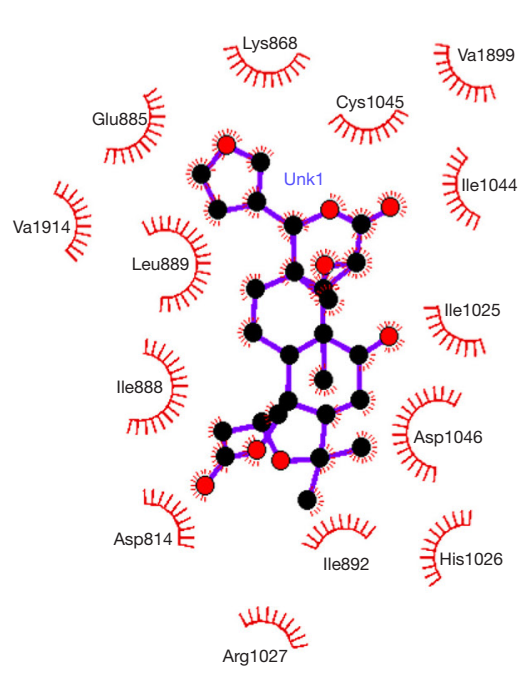

C

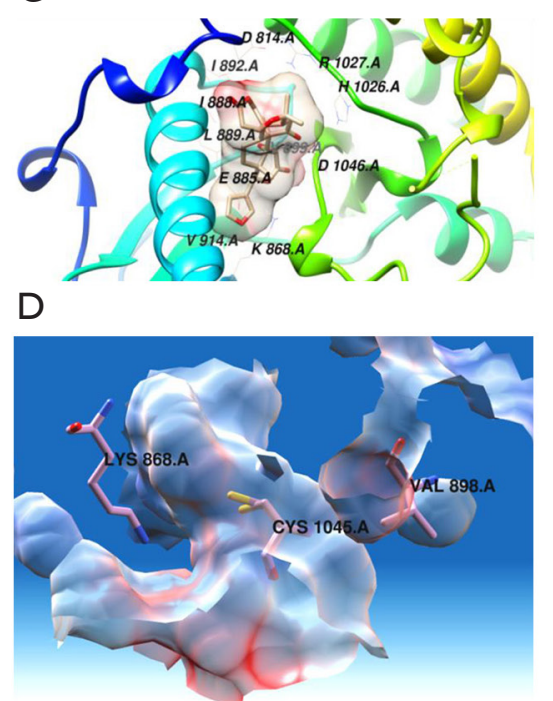

E

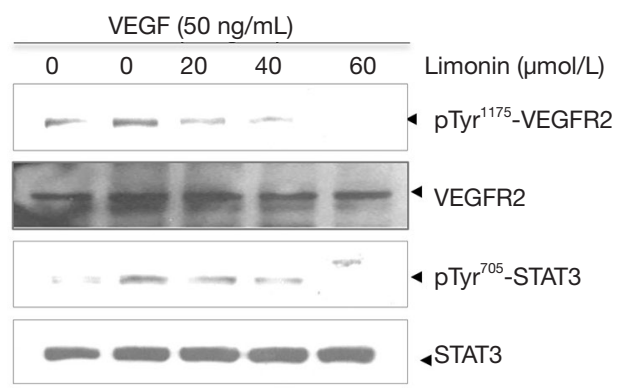

Figure 1 The interaction of limonin with ATP-binding sites of VEGFR2 kinase domain. (A) Limonin's molecular structure. (B) 2-dimensional maps of the interaction between limonin and related amino acids of VEGR2 protein were obtained by using LigPlot software. The types of closely related interactions and bonds. (C) The ribbon structure of green VEGFR2 protein was created by Chimera program (D) ATP binding site of VEGFR2 crystal. (E) Western blotting analysis showed that limonin dose- dependently inhibited the activation of both VEGFR2 $\left(\mathrm{Tyr}^{175}\right)$ and downstream STAT3 $\left(\mathrm{Tyr}^{705}\right)$ triggered by VEGF in endothelial cells.

western blot analysis demonstrated the effects of limonin on VEGFR2 and its downstream signaling pathway proteins in HUVEC cells. VEGF significantly activated VEGFR2 and its downstream targets, while limonin treatment suppressed markedly the VEGF-induced VEGFR-2 phosphorylation at the tyrosine1175 residue, resulting in the reduction of the phosphorylation of STAT3 without affecting its overall protein expression levels (Figure 1E). In summary, these findings indicated that limonin may exert anti-angiogenic effects by directly targeting VEGFR2, inhibiting the binding of ATP to its binding pocket and by antagonizing the VEGFR2-mediated STAT3 signaling cascades.

Limonin inhibits cell migration, capillary-like structure formation in vitro and blocks neovascularization in vivo.
The ability of limonin to inhibit the in vitro angiogenesis of human endothelial cells induced by VEGF were investigated. VEGF increased HUVEC proliferation rate, while higher concentrations of limonin $(240 \mu \mathrm{M})$ displayed only marginal effects on the proliferation of HUVECs and its inhibitory rate was approximately $<50 \%$ (Figure S1A). However, exposure to lower concentrations of limonin at 40 and $60 \mu \mathrm{M}$ significantly inhibited the migration of cells on the scratched area $(\mathrm{P}<0.01)$ following $8-10 \mathrm{~h}$ of VEGF and limonin treatment (Figure $2 A$ ). The migrated number of limonin-treated HUVECs was considerably lower than that of the vehicle control group (Figure $2 B$ ). The Transwell invasion assay further revealed that limonin dosedependently impaired the chemotactic motility of HUVECs 
A

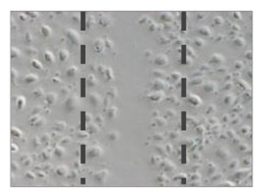

$\mathrm{V}-$

C

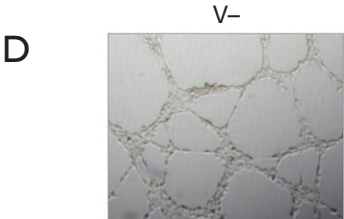

Control

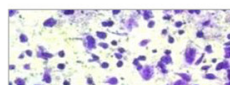

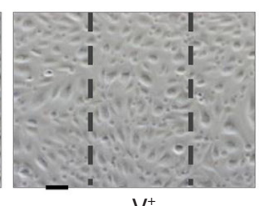

$\mathrm{V}^{+}$

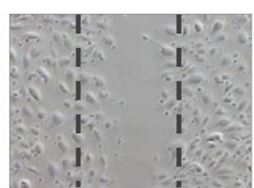
$\mathrm{V}^{+}+40 \mu \mathrm{M}$
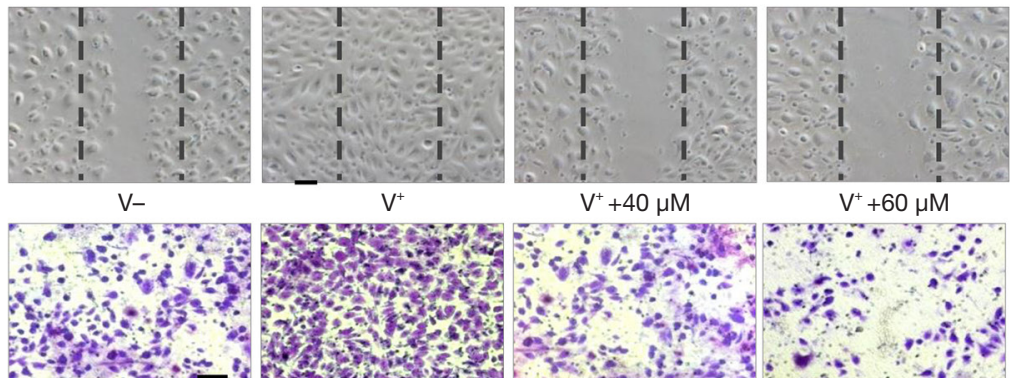

$\mathrm{V}^{+}$

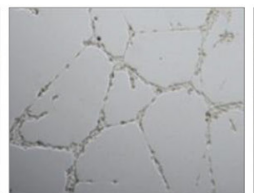

$\mathrm{V}^{+}+40 \mu \mathrm{M}$
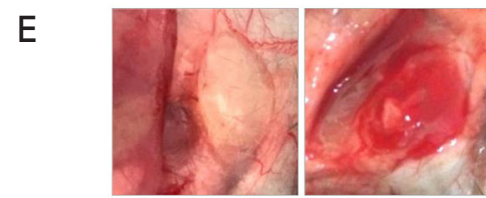

Control

VEGF

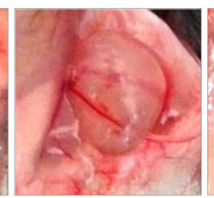

\section{G}

VEGFR2

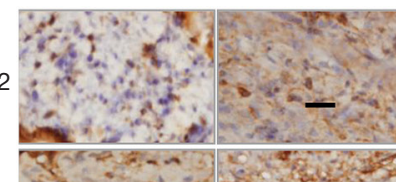

CD31
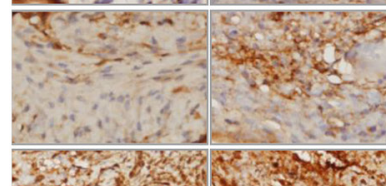

STAT3

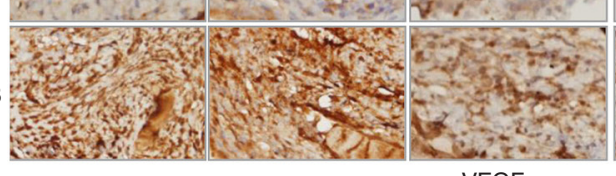

Control

VEGF
VEGF Limonin $200 \mu \mathrm{g}$

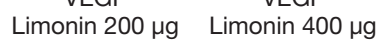

$\mathrm{V}^{+}+60 \mu \mathrm{M}$
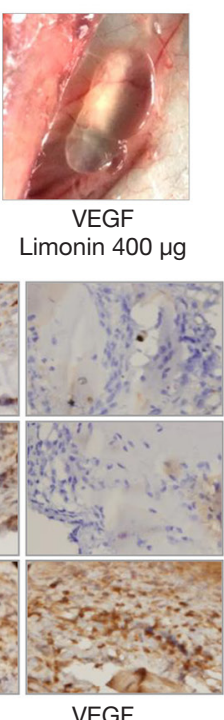

Limonin $200 \mu \mathrm{g} \quad$ Limonin $400 \mu \mathrm{g}$
B

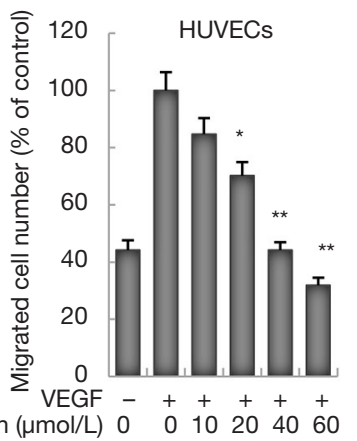

F

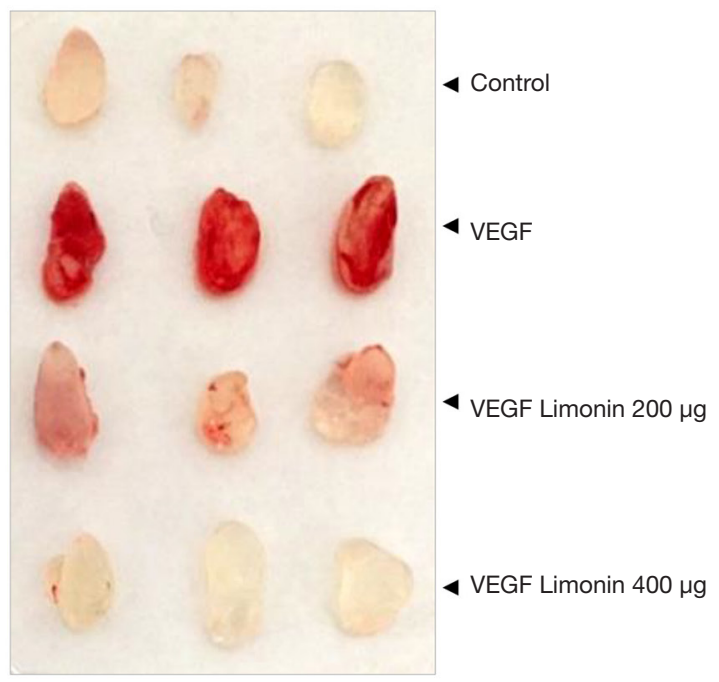

Figure 2 VEGF-induced angiogenesis in vitro and in vivo were inhibited by limonin. (A) VEGF-induced endothelial cells migration was significantly inhibited by limonin in Wound Healing assays, Scale bar=100 $\mu \mathrm{m}$. (B) Quantification of the data from the Wound Healing assays. Scale bar $=100 \mu \mathrm{m}(\mathrm{n}=3)$. ${ }^{*}, \mathrm{P}<0.05$; ${ }^{* *}, \mathrm{P}<0.01$; ${ }^{* *}, \mathrm{P}<0.001$. (C) Limonin inhibited HUVECs cell invasive ability using gelatincoat Boyden inserts. (D) The formation of endothelial cell tubes was inhibited by limonin. Representative fields were displayed in each group $(0.5 \%$ crystal violet staining, magnification at $100 \times)$. Columns, mean from three independent experiments with triplicate. *, $\mathrm{P}<0.05$; **, $\mathrm{P}<0.01$ versus VEGF control. (E,F) Representative matrigel plug images in each group ( $\mathrm{n}=3$ ). (G) Immunohistochemistry was used to analyze the anti-angiogenic effects of limonin on VEGFR2, CD31 and STAT3 on matrigel plug sections (magnification, 400x).

to a similar degree (Figure 2C).

In addition, tube formation of endothelial cells is another key step in vascular sprouting, which controls cell division, migration and the maintenance of cell shape. To characterize the effects of limonin on capillary-like structure formation, HUVECs were grown on matrigel and cultured in the presence of different concentrations of limonin. In the control group, HUVECs could form vessellike structures (Figure 2D). However, exposure to limonin (40 and $60 \mu \mathrm{M}$ ) caused apparent suppression of microvessel sprouting. Therefore, the data suggested that, at the effective concentration of 40 and $60 \mu \mathrm{M}$, limonin strongly inhibited the VEGF-dependent cell migration, invasion, and tubulogenesis of the endothelial cells but not their 
proliferation.

Following these findings, the matrix gel plug experiment was carried out in the mouse angiogenic model to further measure the in vivo anti-angiogenic activity of limonin. The mice treated with limonin (200 or $400 \mu \mathrm{g}$ per plug) inhibited the formation of new blood vessels in matrix plugs compared with those of the normal control group (Figure 2E,F). IHC staining with an anti-CD31 antibody (a specific-endothelial marker) further revealed that limonin treatment strongly reduced the number of CD31-positive endothelial cells inserted into the plug section (Figure 2G). Subsequent analysis of the inhibitory effects of limonin on the VEGFR2-mediated STAT3 signaling pathway was performed by IHC methods. The results indicated that the protein expression levels of VEGFR2 and STAT3 in the plugs with VEGF were increased, whereas those in the limonin-treated plugs were apparently decreased (Figure 2G). These findings were consistent with the in vitro experiment results. These results suggested that limonin could potently inhibit VEGF-induced angiogenesis in vitro and in vivo.

Limonin inhibits migration via the insulin growth factor receptor 1 (IGFR1)/STAT3 signaling in breast cancer cells. The previous findings had confirmed that limonin demonstrated potent inhibitory effects on VEGF-induced angiogenesis in HUVECs via the VEGFR2-mediated STAT3 signaling pathway. Since STAT3 is constitutively and aberrantly activated in the majority of cancer tissues (25), the effects of limonin on the inhibition of STAT3 were examined by transcription factor-binding site (TFBS) enrichment analysis. TFBS analysis revealed that limonin inhibited the expression levels of STAT3 (data not shown) as well as the levels of the majority of the downstream target genes, which were associated with the expression of disease genes in cell-cell interaction, metastasis and angiogenesis according to the MalaCards database (Figure 3A, Table S1). In the present study, the expression levels of two notable target genes, namely insulin growth factor receptor 2 (IGF2) and IGFR1 were significantly downregulated by limonin treatment at 40 and $60 \mu \mathrm{M}$, as shown in the heatmap (Figure S1B). Therefore, the IGF2/IGFR1-mediated STAT3 signaling pathway was selected for further studies.

Western blot analysis indicated that limonin suppressed the phosphorylation of IGFR1 (at tyrosine 1131) with or without IGF2 $(100 \mathrm{ng} / \mathrm{mL})$ and the activation of the downstream target STAT3 (at tyrosine 705) dosedependently. In MDA-MB-231 breast cancer cells, the maximum inhibition was obtained at $60 \mu \mathrm{mol} / \mathrm{L}$
(Figure 3B). In addition, the control experiment included the treatment of the cells with the drug, regorafenib, which is one of the VEGFR2 inhibitors used in the clinic. The data demonstrated that limonin exhibited similar effects with those of regorafenib $(26,27)$ (Figure 3C). However, the total expression levels of these proteins were not significantly affected.

Recent studies have reported that protein tyrosine phosphatase SHP-1 is considered a key negative regulator of STAT3 and is expressed in the majority of cancer cells $(28,29)$. Therefore, the current study investigated whether limonin could regulate the expression of SHP-1 by western blot analysis. Limonin markedly upregulated the expression of SHP-1 at the protein level in a dose-dependent manner (Figure 3C). The effects of limonin on SHP-1 were similar to those of regorafenib. In order to further identify the target of limonin with regard to the inhibition of tumor cell migration, SHP-1 was silenced with specific small interference RNA. The results indicated that silencing of SHP-1 reversed the inhibition of tumor cell migration by limonin (Figure 3D,E), suggesting that SHP-1 is required for inhibiting tumor metastasis by limonin. Moreover, the analyses of the target gene products of STAT3 indicated that limonin preferentially reduced the expression levels of MMP-9 and VEGF at the transcriptional level in a dose-dependent manner, with an effective concentration of approximately $60 \mu \mathrm{M}$ (Figure $3 F$ ). By contrast, the expression levels of the anti-apoptotic and proliferative genes, such as Bcl-2 and Bcl-xL, were not significantly influenced at the same dose of treatment (Figure $3 F$ ). These results were further confirmed at the protein level (Figure $3 G$ ).

Since an association between IGFR1/STAT3 signaling and cancer metastasis has been previously shown (30), the effects of limonin on cancer cell migration were further evaluated using wound healing experiments. The data indicated that following $24 \mathrm{~h}$ of treatment, the control cells almost completely migrated to the initial transparent area, while limonin treatment significantly reduced the migration of MDA-MB-231 and MCF-7 breast cancer cells (Figure $3 H$ ). The data further indicated that the inhibitory activity of limonin on cell migration was not due to its cytotoxic effects, provided that the cell viability was not altered at the concentration ranges tested. The cell growth curves of both breast cancer cell lines were shown in Figure S1C. All the data indicated that limonin could inhibit breast cancer cell migration by blocking the IGFR1/ SHP-1/STAT3 signaling pathway. 
A

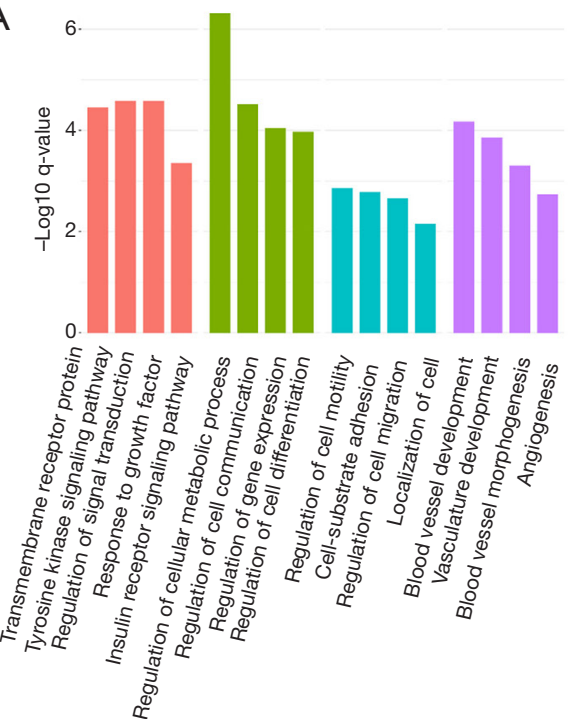

B

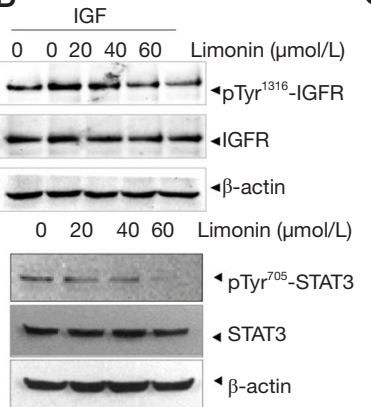

$\begin{array}{lllll}0 & 20 & 40 & 60 & \text { Regorafenib }(\mu \mathrm{mol} / \mathrm{L})\end{array}$

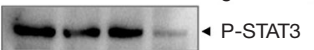

STAT3

C

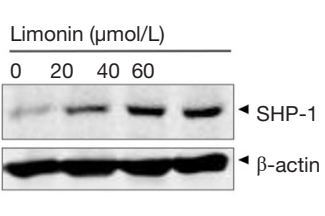

Regorafenib $(\mu \mathrm{mol} / \mathrm{L})$

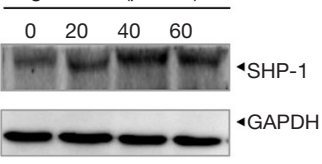

\section{D}

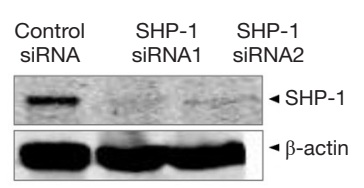

E

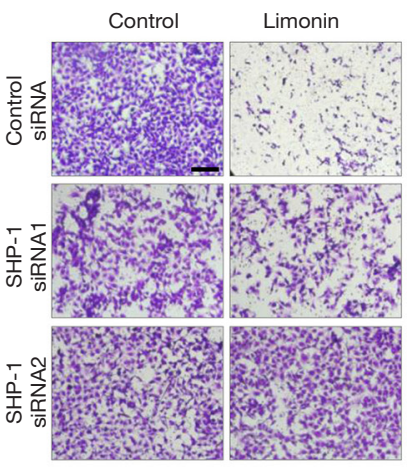

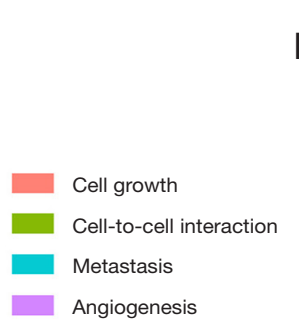

F

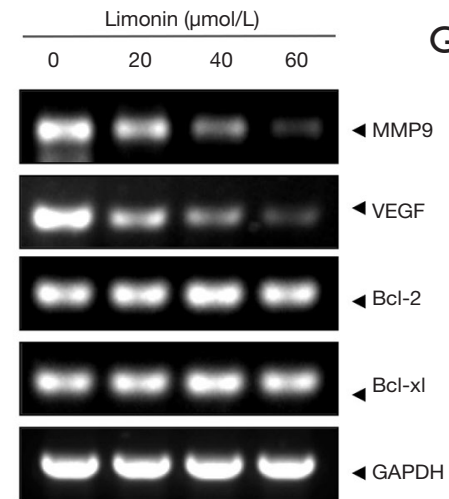

G

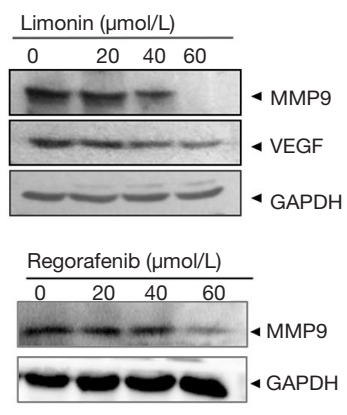

H MDA-MB-23
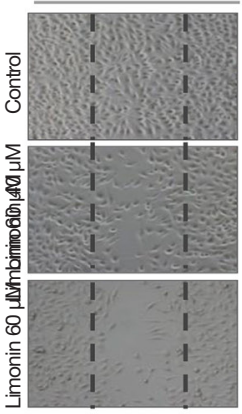

MCF-7

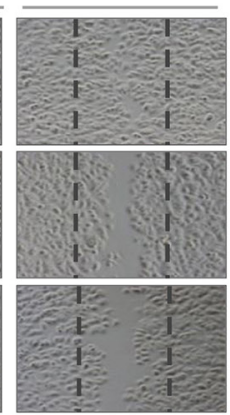

Figure 3 The IGFR1/STAT3 signaling pathway is involved in anti-metastatic effects in limonin treated breast cancer MDA-MB-231 cells. (A) Path enrichment of differentially expressed genes in limonin treated MDA-MB-231 cells. The down-regulated gene subpopulation regulated by the STAT family was used for enrichment analysis. The bar plot showed the highest enrichment score (_log (P value)) for the significant enrichment pathway, the $P$ value cutoff is 0.05 . (B) Limonin and Regorafenib dose-dependently inhibited the phosphorylation of both IGFR1 $\left(\mathrm{Tyr}^{1316}\right)$ and STAT3 $\left(\mathrm{Tyr}^{705}\right)$ in breast cancer cells. (C) Effects of limonin and Regorafenib on SHP-1 in MDA-MB-231 cells were analyzed by Western blotting assay. (D) Immunoblotting assay was used to detect the knockdown efficiency of SHP-1. (E) Transwell migration assay was used to test the inhibitory effect of limonin on MDA-MB-231 cell migration when silencing SHP-1, $0.5 \%$ crystal violet staining, Scale bar $=100 \mu \mathrm{m}$. (F) RT-PCR and (G) Western blotting assays used to test the STAT3-dependent transcriptional activity of MMP9 and VEGF by suppressed of limonin and regorafenib. (H) Wound healing assay was used to examine the inhibition effect of limonin on breast cancer cells migration, Scale bar $=100 \mu \mathrm{m}$.

Limonin inhibits breast cancer invasion via the STAT3 signaling pathway. Following the initial in vitro findings on the effects of limonin on the migration of MDA-MB-231 and MCF-7 cells, our analysis was extended to examine the effects of this compound on lung metastasis of MDAMB-231 cancer cells in two mammary tumor metastasis models as follows: the mouse left ventricle injection tumor metastasis model and the mouse tail vein injection tumor metastasis model. One week prior to tail vein injection, the mice were injected intraperitoneally with vehicle or limonin for 45 days $(31,32)$. The development of lung metastases nearly disappeared in mice that received pretreatment with $25 \mathrm{mg} / \mathrm{kg} /$ day limonin compared with that of the control group. Moreover, the photon flux in the lungs of mice treated at this dose was significantly reduced (Figure 4 A, 95\% CI, $92.97 \%$ to $99.49 \%$; $<<0.001$ ) 
A

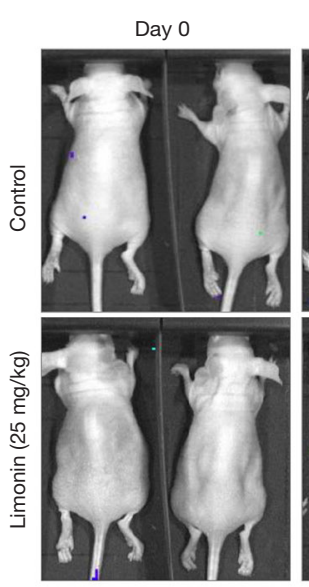

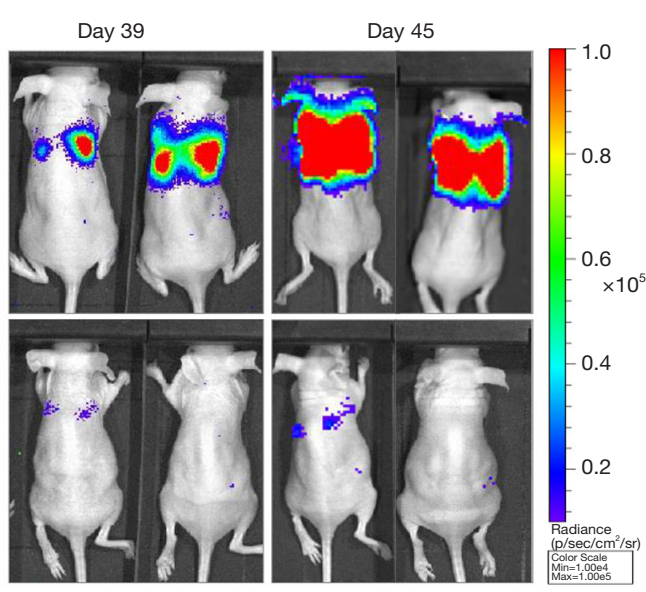

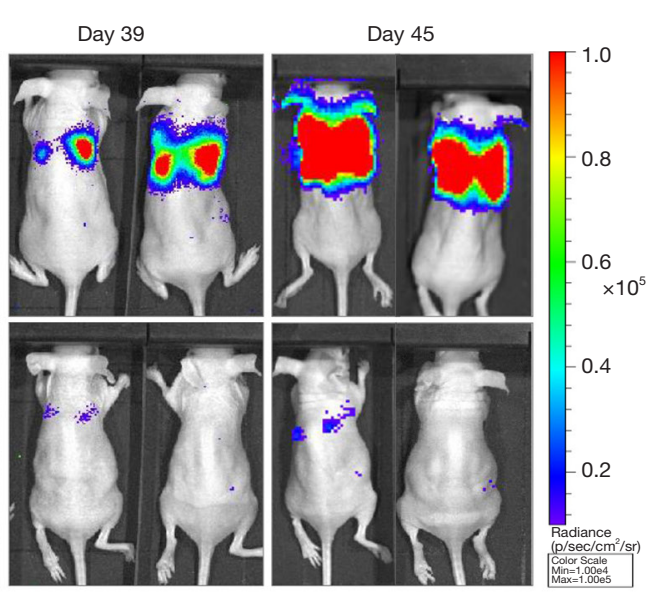

B

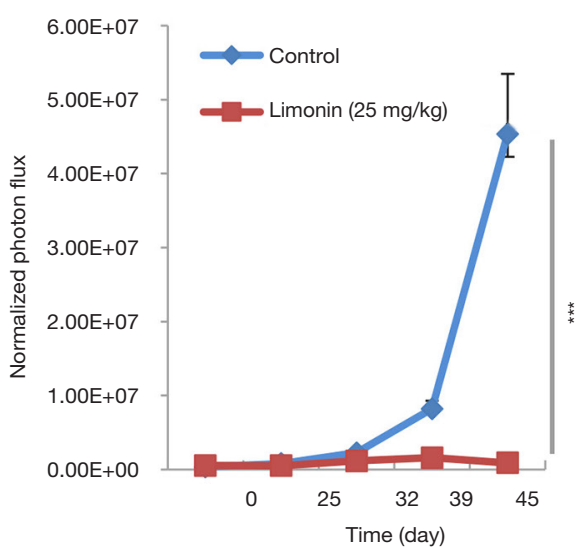

C
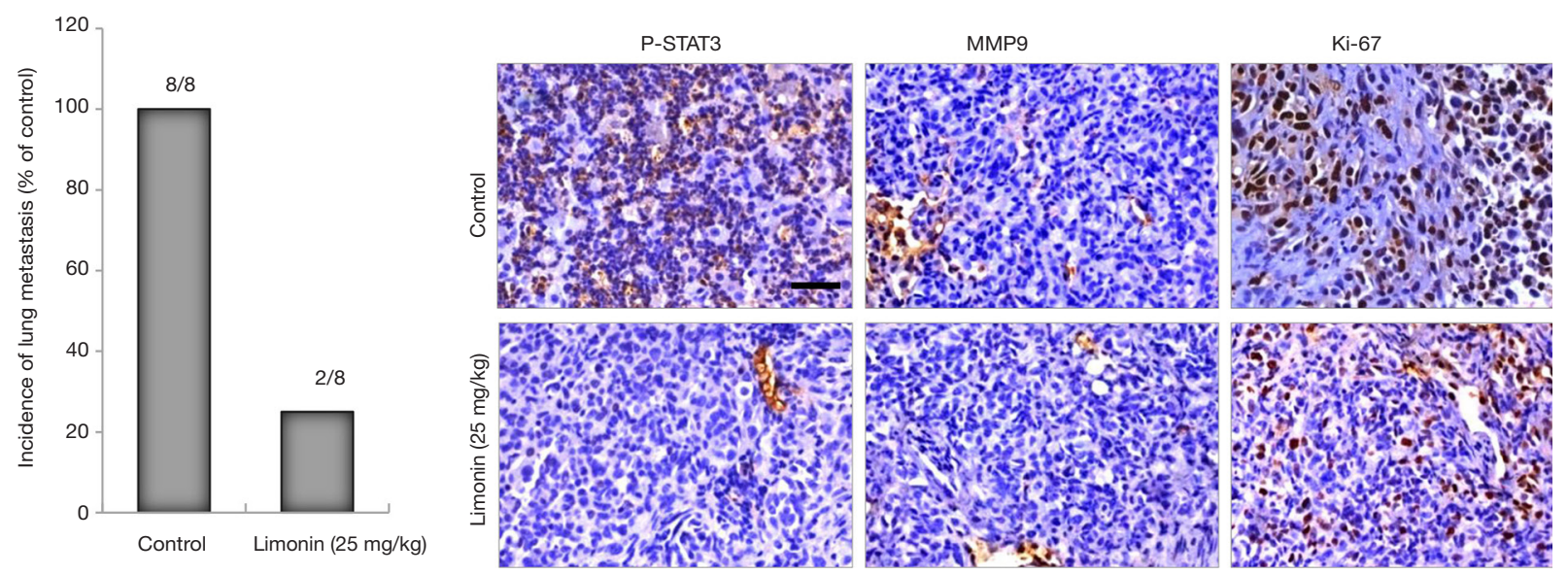

Figure 4 Effect of prevention and treatment of limonin on mouse breast tumor metastasis by tail vein injection. (A) Bioluminescence analysis was used to determine tumor metastasis over a 45-day period. (B) Quantitative analysis of metastatic cells for use in lung bioluminescence analysis, ${ }^{* *}, \mathrm{P}<0.001$. P values were calculated using a two sided Student's $t$ test. $\mathrm{p} / \mathrm{sec} / \mathrm{cm}^{2} / \mathrm{sr}=\mathrm{photons} / \mathrm{second} / \mathrm{cm}^{2} /$ steradian. (C) Quantitative analysis of incidence of lung metastasis. (D) Histological analyses of breast cancer cell lung metastases with administration of limonin or vehicle control (magnification, $400 \times$ ).

compared with that noted in the control mice (Figure 4B). The respective incidence of lung metastasis in the control group and in the limonin $(25 \mathrm{mg} / \mathrm{kg})$ treatment group was $100 \%$ (8 of 8 ) and 25\% (2 of 8), respectively (Figure 4C). To fully validate the possible anti-metastatic mechanisms of limonin in vivo, immunohistochemical (ICH) staining was performed at the end of the treatment. The results revealed that the expression levels of p-STAT3 and MMP9 were clearly decreased in the tumor tissues of mice treated with limonin, while the expression levels of the cell proliferation marker Ki-67 were not altered in the treated groups compared with those of the control mice (Figure 4D).
The mouse left ventricle injection tumor metastasis model was used to further investigate the inhibitory effects of limonin on mammary tumor metastasis. One week prior to the injection of MDA-MB-231 cells, the mice were injected intraperitoneally with vehicle or limonin at $25 \mathrm{mg} / \mathrm{kg} /$ day. The luciferase-labelled MDA-MB-231 mammary tumor cells metastasized to the lung area following 21 days in the control mice (Figure 5A). By contrast, treatment with limonin for 28 days significantly inhibited MDA-MB-231 cell lung metastases in the limonin-treated group (Figure 5A). The images were obtained from the typical metastatic target organs (lung, 
A

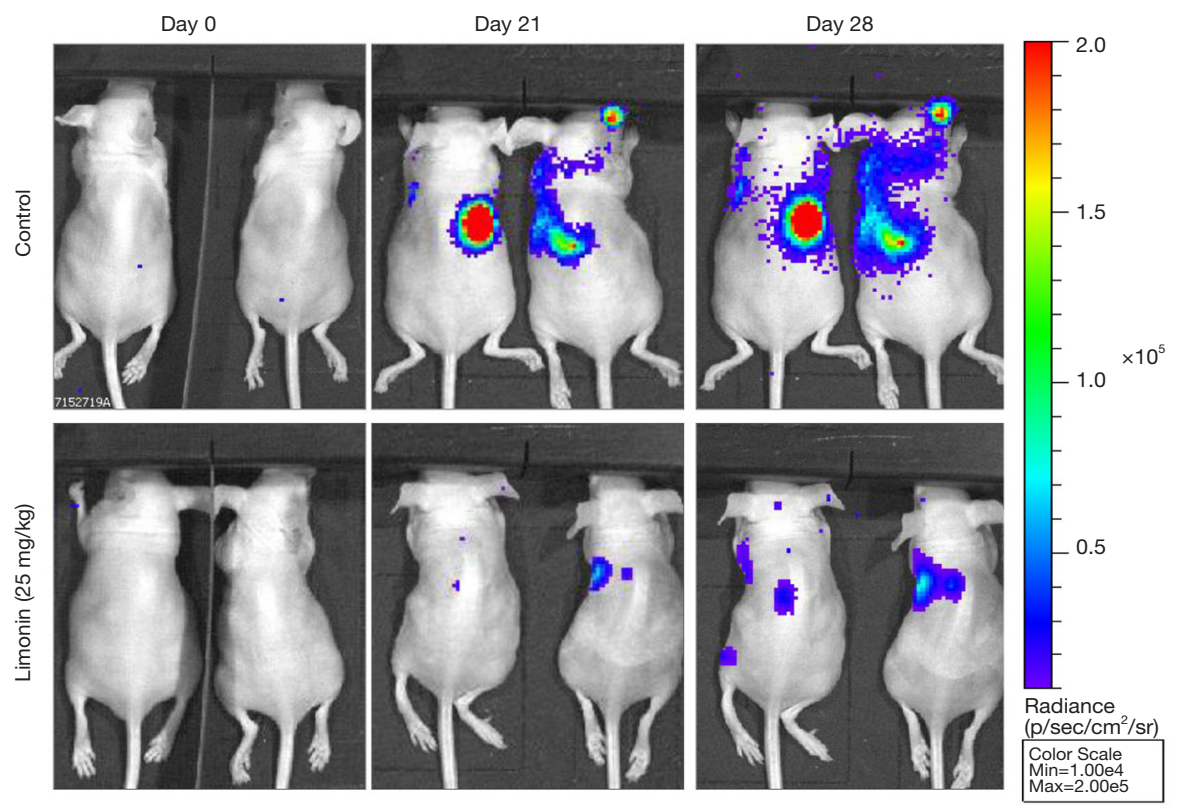

B
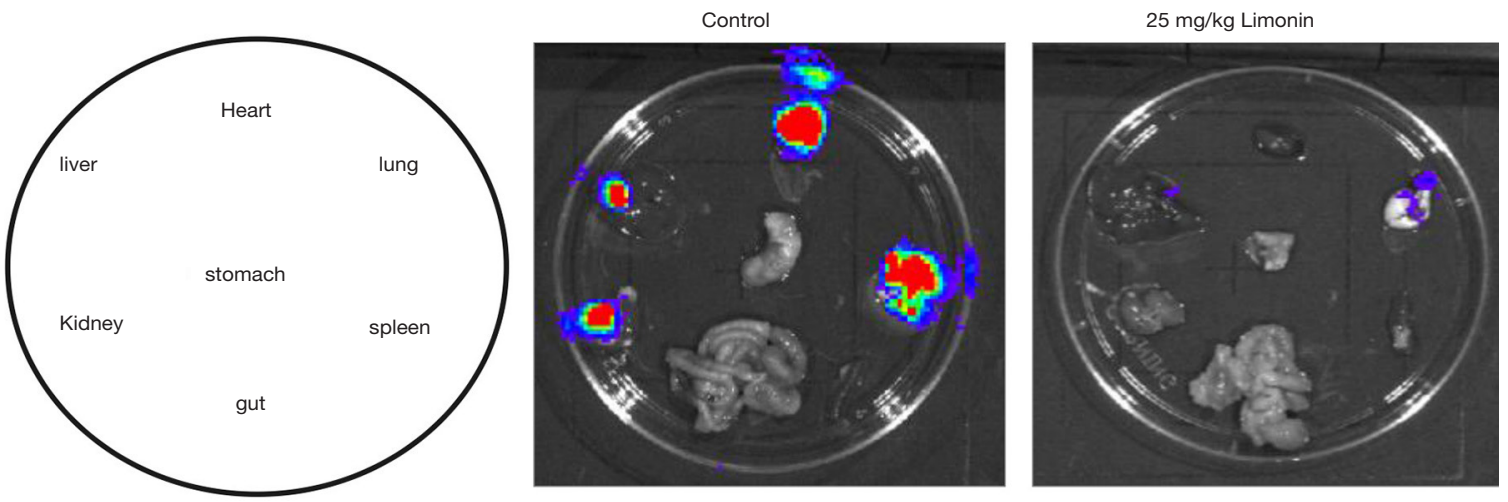

Figure 5 Preventive effects of limonin on mouse breast tumor metastasis by left ventricle injection. (A) Bioluminescence analysis was used to determine the 28-day period tumor metastasis. (B) Limonin prevents metastasis in multiple organs in vivo.

heart, kidney, stomach, spleen, liver and gut) (Figure 5B). The results revealed that limonin treatment significantly reduced the metastatic node numbers in the target organs, indicating that this compound could act in a preventive mode of action.

\section{Discussion}

The prevention and suppression of tumor metastasis and angiogenesis using natural agents may be an effective method for the treatment of metastatic tumors $(6,33)$. In the present study, the anti-metastatic effects of limonin, a natural bioactive molecule in citrus, were investigated. This compound was used at a dose of 40 to $60 \mu \mathrm{M}$ and demonstrated potent inhibitory effects on the migration and formation of capillary structure of the angiogenic vessels. At these concentrations, limonin significantly inhibited the activity of VEGF and of its high-affinity receptor VEGFR2, which are the most widely studied factors in angiogenesis (23). In the present study, computational docking data indicated that the activity patches formed by K868, V899 and D1046 were mapped with the electrostatic 
surface. The furan ring of limonin interacted with the K868 and V899 residues, whereas its alkyl part interacted with D1046 through the hydrophobic interaction. Therefore, this binding mode of limonin to VEGFR2 may prevent the binding of ATP with its active pocket $(18,34)$, leading to the inactivation of several downstream signaling events. The results revealed that limonin could significantly reduce the phosphorylation of VEGFR2 and its downstream target STAT3 in HUVECs induced by VEGF in a dosedependent and time-dependent manner as demonstrated by western blot analysis, indicating that this compound played an anti-angiogenic role mainly through the VEGFR2/ STAT3 signaling pathway.

In addition to the newly discovered anti-angiogenic function of limonin described in the present study, our findings further revealed the anti-metastatic effects of this compound on human breast cancer cells, with the maximum inhibition occurring at 40-60 $\mu \mathrm{M}$. Recently, it was reported that constitutive and aberrant activation of IGFR1 and of the downstream transcription factor STAT3 could play a crucial role in the initiation of cancer transformation and progression (35). The activated form of STAT3 may translocate into the nucleus to regulate the expression of target genes involved in invasion (MMPs) and angiogenesis (VEGF), which participate in the degradation of the basement membrane and the extracellular matrix $(18,34)$. This in turn facilitates cancer cell metastasis and angiogenesis $(36,37)$. Therefore, decreasing the expression levels of MMPs, notably MMP-2 and MMP-9, could in theory inhibit angiogenesis-dependent intravasation and metastasis $(38,39)$. In the present study, the data demonstrated that limonin treatment resulted in the preferential suppression of MMP9 expression, as well as in the downregulation of VEGF expression, at both the protein and mRNA levels. These effects were noted in MDA-MB-231 cells. It has been shown that SHP1 is a critical negative regulator of STAT3 regulation. Silencing SHP-1 markedly reversed the inhibitory activity of limonin on MDA-MB-231 cell motility, indicating that SHP-1 was necessary for limonin-mediated inhibition of tumor invasion. Therefore, it was speculated that the anti-metastatic effects of limonin may be mediated by suppressing the activation of IGFR1/STAT3 and the expression levels of its downstream targets MMP9 and VEGF and by increasing the levels of the negative STAT3 regulator SHP-1 in breast cancer cells. However, further studies are required to validate this hypothesis.

Limonin is one of the major active ingredients of citrus, which has been safely used as a dietary supplement for both nutrition and health-promoting purposes $(40,41)$. In the present study, the data demonstrated that limonin effectively blocked breast cancer angiogenesis and metastasis, at least in part, via the VEGFR2/IGFR1mediated STAT3 signaling pathway. These characteristics render limonin a novel efficient angiogenesis inhibitor that differs from the available compounds used clinically. Limonin may successfully contribute to the prevention and/or delay of the therapeutic resistance noted during single anti-angiogenic therapy. The findings are significant since limited therapeutic options are currently available for highly metastatic breast cancer. Therefore, limonin may be considered a novel candidate agent for the inhibition of tumor invasion, metastasis and angiogenesis that shows promise for future clinical application. However, this experiment also has its limitations. In the two different animal metastasis models, we only give mice intraperitoneal injections without oral or intravenous limonin. The drug metabolism, pharmacokinetics and pharmacodynamics of this compound need to be further studied to determine whether the absorption of limonin is sufficient to function in breast cancer patients.

\section{Acknowledgments}

Funding: This work is supported by the grants from National Natural Science Foundation of China (81260339, 81560485, 816660500), West China Top Class Discripline Project in Basic Medical Sciences, Ningxia Medical University (NXYLXK2017B07 and NXYLXK2017A05); Natural Science Foundation of Ningxia (NZ16142).

\section{Footnote}

Reporting Checklist: The authors have completed the ARRIVE reporting checklist. Available at http://dx.doi. org/10.21037/tcr-20-1992

Data Sharing Statement: Available at http://dx.doi. org/10.21037/tcr-20-1992

Peer Review File: Available at http://dx.doi.org/10.21037/tcr20-1992

Conflicts of Interest: All authors have completed the ICMJE uniform disclosure form (available at http://dx.doi. org/10.21037/tcr-20-1992). The authors have no conflicts 
of interest to declare.

Ethical Statement: The authors are accountable for all aspects of the work in ensuring that questions related to the accuracy or integrity of any part of the work are appropriately investigated and resolved. All mouse experiments were carried out in accordance with the animal experimental regulations approved by the Animal Protection and Utilization Committee of Ningxia Medical University.

Open Access Statement: This is an Open Access article distributed in accordance with the Creative Commons Attribution-NonCommercial-NoDerivs 4.0 International License (CC BY-NC-ND 4.0), which permits the noncommercial replication and distribution of the article with the strict proviso that no changes or edits are made and the original work is properly cited (including links to both the formal publication through the relevant DOI and the license). See: https://creativecommons.org/licenses/by-nc-nd/4.0/.

\section{References}

1. Lambert AW, Pattabiraman DR, Weinberg RA. Emerging Biological Principles of Metastasis. Cell 2017;168:670-91.

2. Mikuła-Pietrasik J, Uruski P, Tykarski A, et al. The peritoneal "soil" for a cancerous "seed": a comprehensive review of the pathogenesis of intraperitoneal cancer metastases. Cell Mol Life Sci 2018;75:509-25.

3. Rankin EB, Giaccia AJ. Hypoxic control of metastasis. Science 2016;352:175-80.

4. Newman DJ, Cragg GM. Natural Products as Sources of New Drugs from 1981 to 2014. J Nat Prod 2016;79:629-61.

5. Maishi N, Hida K. Tumor endothelial cells accelerate tumor metastasis. Cancer Sci 2017;108:1921-6.

6. Naito H, Wakabayashi T, Kidoya H, et al. Endothelial Side Population Cells Contribute to Tumor Angiogenesis and Antiangiogenic Drug Resistance. Cancer Res 2016;76:3200-10.

7. Sinha S, Khan S, Shukla S, et al. Cucurbitacin B inhibits breast cancer metastasis and angiogenesis through VEGFmediated suppression of FAK/MMP-9 signaling axis. Int $\mathbf{J}$ Biochem Cell Biol 2016;77:41-56.

8. Budisan L, Gulei D, Zanoaga OM, et al. Dietary Intervention by Phytochemicals and Their Role in Modulating Coding and Non-Coding Genes in Cancer. Int J Mol Sci 2017;18:1178.
9. Rahman A, Siddiqui SA, Jakhar R, et al. Growth inhibition of various human cancer cell lines by imperatorin and limonin from poncirus trifoliata rafin. Seeds. Anticancer Agents Med Chem 2015;15:236-41.

10. Barreca D, Gattuso G, Bellocco E, et al. Flavanones: Citrus phytochemical with health-promoting properties. Biofactors 2017;43:495-506.

11. Eraky MA, El-Kholy AA, Rashed GA, et al. Dose-response relationship in Schistosoma mansoni juvenile and adult stages following limonin treatment in experimentally infected mice. Parasitol Res 2016;115:4045-54.

12. Gualdani R, Cavalluzzi MM, Lentini G, et al. The Chemistry and Pharmacology of Citrus Limonoids. Molecules 2016;21:245-9.

13. Hu M, Peng S, He Y, et al. Lycorine is a novel inhibitor of the growth and metastasis of hormone-refractory prostate cancer. Oncotarget 2015;6:15348-61.

14. Tundis R, Loizzo MR, Menichini F. An overview on chemical aspects and potential health benefits of limonoids and their derivatives. Crit Rev Food Sci Nutr 2014;54:225-50.

15. Akihisa T, Yokokawa S, Ogihara E, et al. MelanogenesisInhibitory and Cytotoxic Activities of Limonoids, Alkaloids, and Phenolic Compounds from Phellodendron amurense Bark. Chem Biodivers 2017;14:145-67.

16. Chidambara Murthy KN, Jayaprakasha GK, Patil BS. Citrus limonoids and curcumin additively inhibit human colon cancer cells. Food Funct 2013;4:803-10.

17. Chen J, Wang J, Lin L, et al. Inhibition of STAT3 signaling pathway by nitidine chloride suppressed the angiogenesis and growth of human gastric cancer. Mol Cancer Ther 2012;11:277-87.

18. Li T, Liu X, Shen Q, et al. Salinomycin exerts antiangiogenic and anti-tumorigenic activities by inhibiting vascular endothelial growth factor receptor 2-mediated angiogenesis. Oncotarget 2016;7:26580-92.

19. Morris GM, Huey R, Lindstrom W, et al. AutoDock4 and AutoDockTools4: Automated docking with selective receptor flexibility. J Comput Chem 2009;30:2785-91.

20. O'Boyle NM, Banck M, James CA, et al. Open Babel: An open chemical toolbox. J Cheminform 2011;3:33.

21. Romero-Garmendia I, Garcia-Etxebarria K, HernandezVargas H, et al. Transcription Factor Binding Site Enrichment Analysis in Co-Expression Modules in Celiac Disease. Genes (Basel) 2018;9:234-47.

22. Wang J, Zhang L, Chen G, et al. Small molecule 1 '-acetoxychavicol acetate suppresses breast tumor metastasis by regulating the SHP-1/STAT3/ 
MMPs signaling pathway. Breast Cancer Res Treat 2014;148:279-89.

23. Ferrara N, Gerber HP, LeCouter J. The biology of VEGF and its receptors. Nat Med 2003;9:669-76.

24. Dong Y, Lu B, Zhang X, et al. Cucurbitacin E, a tetracyclic triterpenes compound from Chinese medicine, inhibits tumor angiogenesis through VEGFR2mediated Jak2-STAT3 signaling pathway. Carcinogenesis 2010;31:2097-104.

25. Ma JH, Qin L, Li X. Role of STAT3 signaling pathway in breast cancer. Cell Commun Signal 2020;18:33.

26. Refolo MG, D'Alessandro R, Lippolis C, et al. IGF$1 \mathrm{R}$ tyrosine kinase inhibitors and Vitamin $\mathrm{K} 1$ enhance the antitumor effects of Regorafenib in HCC cell lines. Oncotarget 2017;8:103465-76.

27. D'Alessandro R, Refolo MG, Lippolis C, et al. Antagonism of sorafenib and regorafenib actions by platelet factors in hepatocellular carcinoma cell lines. BMC Cancer 2014;14:351.

28. Saraswati S, Alhaider A, Abdelgadir AM, et al. Phloretin attenuates STAT-3 activity and overcomes sorafenib resistance targeting SHP-1-mediated inhibition of STAT3 and Akt/VEGFR2 pathway in hepatocellular carcinoma. Cell Commun Signal 2019;17:127.

29. Koh JS, Joo MK, Park JJ, et al. Inhibition of STAT3 in gastric cancer: role of pantoprazole as SHP-1 inducer. Cell Biosci 2018;8:50.

30. Rho O, Kim DJ, Kiguchi K, et al. Growth factor signaling pathways as targets for prevention of epithelial carcinogenesis. Mol Carcinog 2011;50:264-79.

31. Kim J, Jayaprakasha GK, Patil BS. Limonoids and their anti-proliferative and anti-aromatase properties in human breast cancer cells. Food Funct 2013;4:258-65.

Cite this article as: Chen J, Liu BX, Shen Q, Li N, Ling J, Xiao M, Jiao HY, Li T. Limonin inhibits angiogenesis and metastasis of human breast cancer cells by suppressing the VEGFR2/IGFR1-mediated STAT3 signaling pathway. Transl Cancer Res 2020;9(11):6820-6832. doi: 10.21037/tcr-20-1992
32. Fang Y, Chen Y, Yu L, et al. Inhibition of breast cancer metastases by a novel inhibitor of TGFbeta receptor 1.J Natl Cancer Inst 2013;105:47-58.

33. Safe S, Kasiappan R. Natural Products as Mechanismbased Anticancer Agents: Sp Transcription Factors as Targets. Phytother Res 2016;30:1723-32.

34. Mendes RT, Nguyen D, Stephens D, et al. Hypoxiainduced endothelial cell responses - possible roles during periodontal disease. Clin Exp Dent Res 2018;4:241-8.

35. Gariboldi MB, Ravizza R, Monti E. The IGFR1 inhibitor NVP-AEW541 disrupts a pro-survival and pro-angiogenic IGF-STAT3-HIF1 pathway in human glioblastoma cells. Biochem Pharmacol 2010;80:455-62.

36. Sakamoto KM, Grant S, Saleiro D, et al. Targeting novel signaling pathways for resistant acute myeloid leukemia. Mol Genet Metab 2015;114:397-402.

37. Shen F, Kirmani KZ, Xiao Z, et al. Nuclear protein isoforms: implications for cancer diagnosis and therapy. J Cell Biochem 2011;112:756-60.

38. Foda HD, Zucker S. Matrix metalloproteinases in cancer invasion, metastasis and angiogenesis. Drug Discov Today 2001;6:478-82.

39. Salimi Sartakhti J, Manshaei MH, Sadeghi M. MMP-TIMP interactions in cancer invasion: An evolutionary game-theoretical framework. J Theor Biol 2017;412:17-26.

40. Marlett JA, Cheung TF. Database and quick methods of assessing typical dietary fiber intakes using data for 228 commonly consumed foods. J Am Diet Assoc 1997;97:1139-48, 1151; quiz 1149-50.

41. Lv X, Zhao S, Ning Z, et al. Citrus fruits as a treasure trove of active natural metabolites that potentially provide benefits for human health. Chem Cent J 2015;9:68. 